In this paper I have concentrated on functions of one variable, but the remarks apply with greater force to partial differential equations, particularly in the desirability, both for the sake of accuracy and a minimum of labour, of using conveniently large intervals.

National Physical Laboratory

L. Fox

Teddington, England

and NBSCL

${ }_{1}$ Numerical Methods of Analysis in Engineering. Edited by L. E. Grinter, Macmillan Company, New York, 1949, chapter 4.

${ }^{2} \mathrm{~L}$. Fox, "Some improvements in the use of relaxation methods for the solution of ordinary and partial differential equations," Royal Soc., London, Proc., v. 190A, 1947, p. 31-59.

${ }^{3}$ L. Fox \& J. C. P. Miller, "Table-making for large arguments. The exponential integral," MTAC, v. 4, 1951, p. 163-167. p. 3-11.

"See, for example, J. C. P. Miller, "Checking by differences-I," MTAC, v. 4, 1950,

J. Tond, "The conditions of a certain matrix," Cambridge Phil. Soc., Proc., v. 46, 1950, p. 116-118.

\title{
Monte Carlo Matrix Inversion and Recurrent Events
}

1. Introduction. Recently WAsow ${ }^{1}$ has given a necessary and sufficient condition that one of two unbiased estimators of the inverse element of a given matrix has a smaller variance. Using the theory of recurrent events, we extend a result by FELLER ${ }^{2}$ in order to generalize and reinterpret Wasow's condition. To do this let us consider a simple discrete Markov process with a finite number of states. Of these $m+1$ states denoted by $0,1,2, \cdots, m$, we prescribe that the state named " 0 " is the only death state or sink-in the sense that the random walk ends when this state is reached. Let $p_{i k}$ be the one-step transition probabilities for $i, k=1, \cdots, m$, and let

$$
p_{i 0}=1-\sum_{k=1}^{m} p_{i k} \quad \text { for } \quad i=1, \cdots, m .
$$

Further, we assume that each $p_{i 0}>0$. When $n>0$ define $p_{i k}^{(n)}$ as the probability that in a random walk starting at state $i$, state $k$ is visited on the $n$-th step; and define $p_{i k}^{(0)}=\delta_{i k}$, where $\delta_{i k}$ is the Kronecker delta.

Now if $\left(q_{i k}\right)=\left(\delta_{i k}-p_{i k}\right)$ is the $m$ by $m$ matrix whose inverse $\left(q^{i k}\right)$ is desired, we may estimate each element $q^{i k}$ by Monte Carlo methods as in WASOW ${ }^{2}$, FORSYTHE \& LEIBLER ${ }^{3}$, and CURTISS ${ }^{4}$, since

$$
q^{i k}=\sum_{n=0}^{\infty} p_{i \mathfrak{k}}^{(n)}
$$

Two estimators have been investigated. The first estimator is the sample mean of the random variable $s_{i k}$ where $s_{i k}=p_{\mathbf{k}_{0}}^{-1}$ if the random walk having started at state $i$ stops just after visiting state $k$, and $s_{i k}=0$ if otherwise. The second estimator is the sample mean of the random variable $v_{i k}$ where $v_{i k}$ is the number of visits to state $k$ in a random walk having started at 
state $i$. Now it is known that both these estimators are unbiased, that is,

$$
E\left(s_{i k}\right)=q^{i k}
$$

and

$$
E\left(v_{i k}\right)=q^{i k}
$$

where $E$ is the expected value operator. Further, the second moments of these random variables can be expressed directly in terms of the $q^{i k}$ and $p_{k 0}$. We have

$$
E\left(s_{i k}^{2}\right)=p_{k 0}^{-1} q^{i k}
$$

and

$$
E\left(v_{i k}^{2}\right)=\left(2 q^{k k}-1\right) q^{i k} .
$$

Relations (1) and (3) were proved by Forsythe \& LEIBLER ${ }^{3}$, and relations (2) and (4) were derived independently by CURTISs ${ }^{4}$ and by the author. Now, Wasow ${ }^{1}$ showed that

$$
E\left(s_{i k}^{2}\right)=\frac{\lambda_{i k}}{p_{k 0}\left(1-\lambda_{k k}\right)} \text { for } i \neq k
$$

and

$$
E\left(v_{i k}^{2}\right)=\frac{\lambda_{i k}\left(1+\lambda_{k k}\right)}{\left(1-\lambda_{k k}\right)^{2}} \text { for } i \neq k
$$

where he defined $\lambda_{i k}$ as "the probability of going from state $i$ to state $k$ without passing through state $k$ on the way; i.e., $\lambda_{i k}$ is the total probability associated with all paths connecting state $i$ and state $k$, all intermediate points being different from state $k$." Thus, we have before us two different interpretations of the second moments of $s_{i k}$ and $v_{i k}$ in the case $i \neq k$.

2. Recurrent Events. Using the concept of recurrent event as developed by Feller ${ }^{2}$ we now extend a result of Feller ${ }^{2}$ to establish a general theorem which shows the fundamental probabilistic relation between the two interpretations for the moments. When $n>0$ define $r_{i 2}^{(n)}$ as the probability that in a random walk starting at state $i$, state $k$ is visited for the first time on the $n$-th step, and define $r_{i k}^{(0)}=0$. Let

$$
r_{i k}=\sum_{n=0}^{\infty} r_{i k}^{(n)}
$$

so that $r_{i k}$ equals the probability that in a random walk starting at state $i$ state $k$ is eventually visited.

TheOREM: $q^{i k}-\delta_{i k}=r_{i k} q^{k k}$.

Proof: By considering the mutually exclusive and exhaustive cases it follows that

$$
p_{i k}^{(n)}=r_{i k}^{(n)}+r_{i k}^{(n-1)} p_{k \mathfrak{k}}^{(1)}+\cdots+r_{i k}^{(1)} p_{k \mathfrak{k}}^{(n-1)} \text { for } n>0
$$


and since $r_{i k}^{(0)}=0$ and $p_{i k}^{(0)}=\delta_{i k}$ that

$$
p_{i k}^{(n)}=\sum_{j=0}^{n} r_{i k}^{(n-j)} p_{i k}^{(j)} \text { for } n>0 .
$$

Next we define

$$
P_{i k}(i)=\sum_{n=0}^{\infty} p_{i k}^{(n)} u^{n}
$$

as the probability generating function for the sequence $\left\{p_{i k}^{(n)}\right\}$, and define

$$
R_{i k}(u)=\sum_{n=0}^{\infty} r_{i \mathfrak{z}}^{(n)} u^{n}
$$

as the probability generating function for the sequence $\left\{r_{i k}^{(n)}\right\}$. Hence by a basic property of generating functions we have by $\left({ }^{*}\right)$

$$
P_{i k}(u)-\delta_{i k}=R_{i k}(u) P_{k k}(u) \text { for all } u .
$$

Putting $u=1$ we get

However, this is

$$
\sum_{n=0}^{\infty} p_{i k}^{(n)}-\delta_{i k}=\left(\sum_{n=0}^{\infty} r_{i k}^{(n)}\right)\left(\sum_{n=0}^{\infty} p_{k i k}^{(n)}\right) .
$$

which was to be proved.

$$
q^{i k}-\delta_{i k}=r_{i k} q^{k k}
$$

As corollaries we get the explicit relations

$$
r_{i k}=\frac{q^{i k}-\delta_{i k}}{q^{k k}}
$$

and

since by definition

$$
q^{i k}=\frac{r_{i k}^{1-\delta_{i k}}}{1-r_{k k}}
$$

and by assumption

$$
q^{k k} \geq p_{k \mathfrak{k}}^{(0)}=1>0
$$

$$
1-r_{k k} \geq p_{k 0}>0 \text {. }
$$

Thus we have a direct derivation of the general relation between $q^{i k}$ and $r_{i k}$ for all $i$ and $k$.

As a matter of interest, while working with Wasow, the author first derived relations (5) and (6) indirectly by equating expression (3) to $\left(3^{\prime}\right)$, and expression (4) to $\left(4^{\prime}\right)$. Thus the relation of $q^{i k}$ to $\lambda_{i k}$ was found. Next it was seen that $\lambda_{i k}$ is, in fact, just $r_{i k}$. An informal verification is as follows.

The probability that a random walk initially traverses a given path is equal to the probability that the walk takes that path times the probability that the walk thereafter follows some path from the class of possible paths remaining. But the latter probability is one. Therefore $\lambda_{i k}$ may be replaced by $r_{i k}$.

We may now write the second moments of our two random variables in terms of $r_{i k}$.

$$
E\left(s_{i k}^{2}\right)=\frac{1}{p_{k 0}} \frac{r_{i \mathfrak{k}}^{1-\delta_{i k}}}{1-r_{k k}}
$$


and

$$
E\left(v_{i k}^{2}\right)=\frac{1+r_{k k}}{1-r_{k k}} \frac{r_{i k^{0}}^{1-\delta_{i k}}}{1-r_{k k}} .
$$

Thus, a comparison of (3) and (4) yields

$$
E\left(s_{i k}^{2}\right)<E\left(v_{i k}^{2}\right) \text { if and only if } \frac{1}{p_{k 0}}<2 q^{k k}-1
$$

and similarly, (7) and (8) give

$$
E\left(s_{i z}^{2}\right)<E\left(v_{i z}^{2}\right) \text { if and only if } \frac{1}{p_{k 0}}<\frac{1+r_{k k}}{1-r_{k k}} \text {. }
$$

This, of course, is Wasow's result expressed in terms of $r_{k k}$ rather than $\lambda_{k k}$. Since $r_{k k}$ is usually unknown, but $r_{k k} \geqslant p_{k k} ;(10)$ implies

$$
E\left(s_{i k}^{2}\right)<E\left(v_{i k}^{2}\right) \text { if } \frac{1}{p_{k 0}}<\frac{1+p_{k k}}{1-p_{k k}} .
$$

These recurrent events associated with this stochastic process have permitted the derivation of further results which will be treated in a subsequent paper.

University of California

H. P. Edmundson at Los Angeles

${ }^{1} \mathrm{~W}$. R. WASOW, "A note on the inversion of matrices by random walks," MTAC, v. 6, 1952, p. 78-81.

${ }^{2}$ W. FELLER, An Introduction to Probability Theory and its Applications, v. 1, New York, 1950.

3'G. E. Forsythe \& R. A. Leibler, "Matrix inversion by a Monte Carlo method," $M T A C$, v. 4, 1950, p. 127-129.

4 J. H. CuRTISS, "Sampling methods applied to differential and difference equations," IBM Seminar on Scientific Computations, November 1949.

\section{RECENT MATHEMATICAL TABLES}

1042[A].-C. E. Fröberg, Hexadecimal Conversion Tables. Lund, 1952, 20 p., $21.7 \times 14.6 \mathrm{~cm}$.

These tables are designed to assist in the coding of problems for binary computers. There are four tables. Table 1 is a table of the integers $1(1) 2^{10}\left(2^{4}\right) 2^{12}$ in both decimal and hexadecimal notation. Table 2 gives the hexadecimal equivalents of $n \cdot 10^{-2 k}, n=1(1) 100, k=1(1) 8$, correct to 13 hexadecimals. Table 3 gives the hexadecimal equivalent of 50 frequently used constants correct to 16 hexadecimals. Finally Table 4 gives the decimal equivalents of numbers of the form

$$
n \cdot 16^{-k} \quad n=1(1) 15, k=1(1) 13 .
$$

Results are given to $16 \mathrm{D}$. The letters A B C D E F are used as hexadecimal notation for the numbers ten through fifteen. A few examples of the use of the tables are given in the introduction. This handy booklet should find its way into many a coding room.

D. H. L. 